\title{
Forecasting hospital pneumonia admissions using influenza surveillance, climate and community data
}

\author{
Joshua Wong ${ }^{1}$, Hanley Ho${ }^{1}$, Angela Chow ${ }^{1,} 2$ \\ ${ }^{1}$ Department of Clinical Epidemiology,Tan Tock Seng Hospital, Singapore, Singapore, ${ }^{2}$ Saw Swee Hock School of Public Health, National University \\ of Singapore, Singapore, Singapore \\ Objective
}

Using the information that we have available, our primary objective is to explore if there was any cross-correlation between pneumonia admissions and hospital influenza positivity. We then aim to develop a data driven approach to forecast pneumonia admissions using data from our hospital's weekly surveillance. We also attempted using external sources of information such as national infectious diseases notifications and climate data to see if they were useful for our model.

\section{Introduction}

Influenza peaks around June and December in Singapore every year. Facing an ageing population, hospitals in Singapore have been constantly reaching maximum bed occupancy. The ability to be able to make early decisions during peak periods is important. Tan Tock Seng Hospital is the second largest adult acute care general hospital in Singapore. Pneumonia-related emergency department (ED) admissions are a huge burden to the hospital's resources. The number of cases vary year on year as it depends on seasonal vaccine effectiveness and the population's immunity to the circulating strain. While many pneumonia cases are of unknown origin, they tend to mirror the influenza seasons very closely.

\section{Methods}

We used data from epidemiological week (e-week) 1 of 2013 to e-week 34 of August 2017 to train our model, with the next 52 weeks (e-week of 35 of 2017 to e-week 34 of 2018) being used as validation cohort. Pneumonia and influenza data were obtained from our hospital's weekly surveillance. National level acute upper respiratory illness (AURI) was obtained from Ministry of Health's (MOH) weekly infectious diseases bulletin. Climate data were obtained from the National Environment Agency's website. Daily rainfall, temperature and wind data from the S20 satellite station were used. Automatic autoregressive (A-ARIMA), nonseasonal and seasonal vector autoregressive models (VAR) were used to either analyse the univariate pneumonia trends or simultaneously model pneumonia, influenza, AURI notification and climatic data. Granger-causality tests were performed to check if these variables were causal of pneumonia admissions. As most of the seasonal variation are seen in older patients, stratified analysis were performed on those that were below and above 65 years old. Forecasts were calculated up to 3 weeks in advance. Mean absolute error (MAE), mean absolute percentage error (MAPE), and root mean squared error (RMSE) were used to validate the model performance. These performance metrics were applied on 3-week ahead forecasts comparing A-ARIMA, VAR, and seasonal-adjusted VAR.

\section{Results}

Figure 1 shows that both influenza and pneumonia admissions follow similar trends. We see that the number of influenza cases have reduced as compared to the previous years. The number hospital influenza cases and the number of AURI cases nationwide are strongly cross-correlated with pneumonia admissions. Granger-causality tests confirmed the directionality of the relationships ( $\mathrm{p}<0.01$ ). Climate factors do not strongly affect the number of pneumonia admissions. (Fig 2) Unsurprisingly, the A-ARIMA model showed that the 1-day forecasts were most accurate (MAE: 7.0; MAPE: 12.7; RMSE: 8.7 for elderly subgroup). However, the 3-day ahead forecasts were only slightly less precise (MAE: 7.2 ; MAPE: 13.2; RMSE: 9 for elderly subgroup). Testing for significant lags using the various information criteria suggested that a lag3 model should be used. The non-seasonal and seasonal VAR models showed that historical pneumonia admissions and influenza positivity was the best model. The MAPE for all 3 models hovered between $12-13 \%$, with the A-ARIMA model performing slightly better. This is not surprising as the A-ARIMA takes the latest information at hand to derive the best model. Accounting for seasonality allowed better precision as compared to the nonseasonal VAR but was not better as compared to the A-ARIMA model. 


\section{Conclusions}

Hospital surveillance data are the most useful for developing forecast models for hospital pneumonia admissions. Climate data were likely not to be useful as Singapore does not experience much variaton in weather throughout the year. Pneumonia peaks do not follow necessarily fall on the same week every season. Therefore, both the autoregressive and seasonal-adjusted vector autoregressive models can be useful complements to each other for forecasting pneumonia admissions.

Fig 1: Weekly trend for hospital influenza and pneumonia admissions

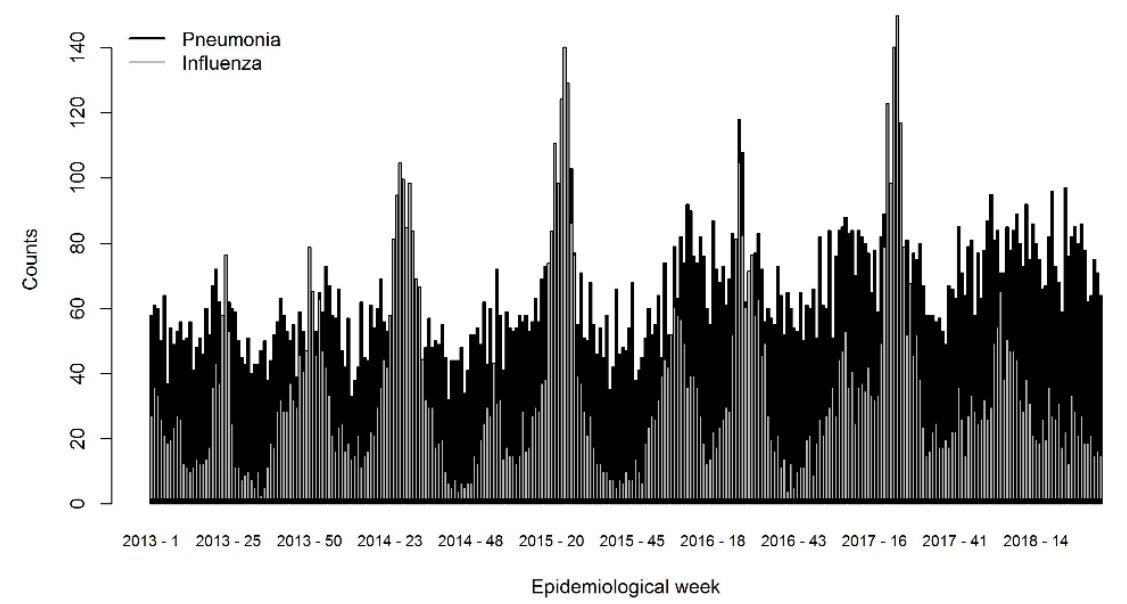

Fig 2: Cross-correlations between pneumonia admissions and various factors
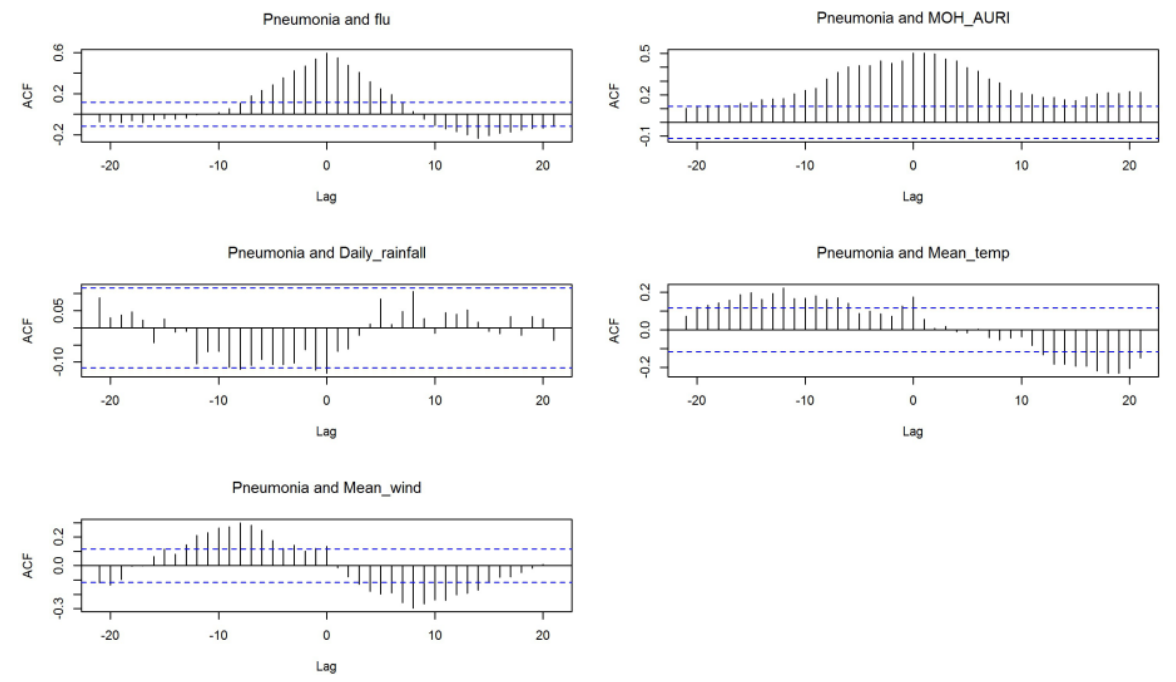

ISDS Annual Conference Proceedings 2019. This is an Open Access article distributed under the terms of the Creative Commons AttributionNoncommercial 4.0 Unported License (http://creativecommons.org/licenses/by-nc/3.0/), permitting all non-commercial use, distribution, and reproduction in any medium, provided the original work is properly cited. 
Fig 3: Forecasted values for the A-ARIMA model
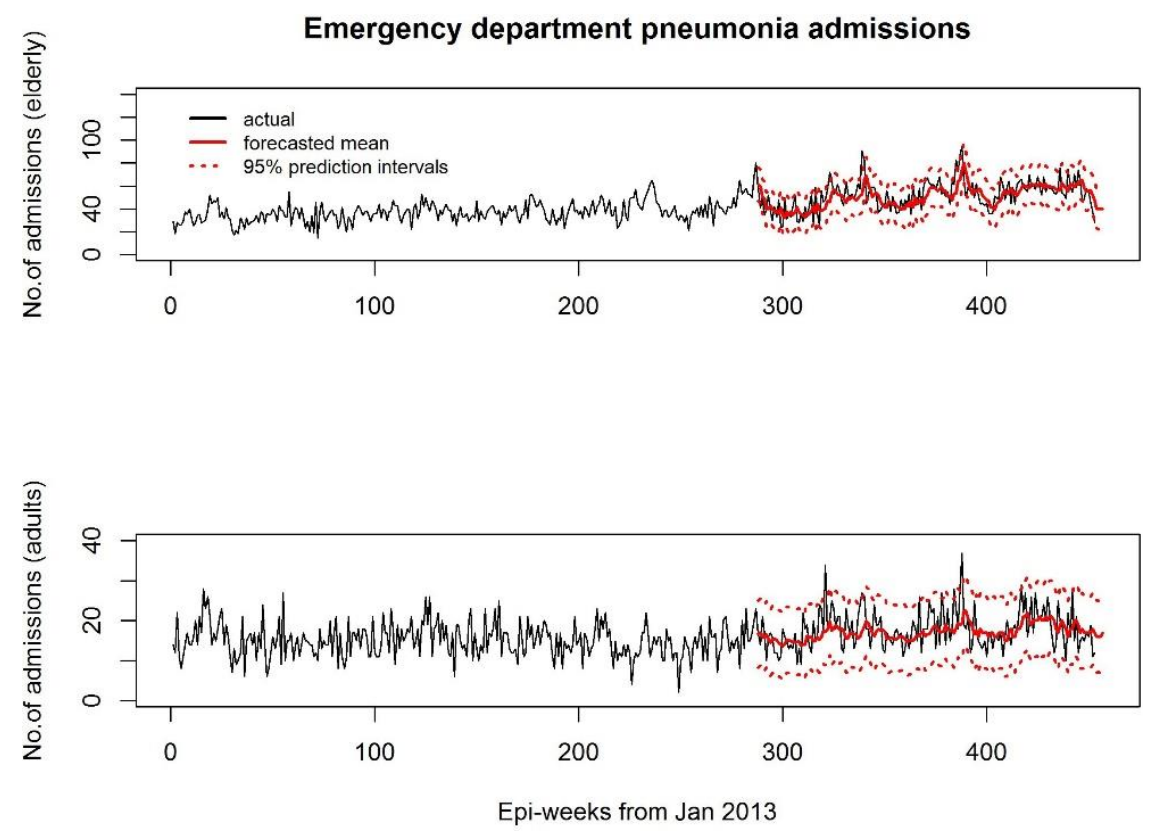

Table 1: Stratified A-ARIMA lag1 to lag4 models.

\begin{tabular}{|c|c|c|c|c|c|c|c|c|}
\hline & E_t-1 & E_t-2 & E_t-3 & E_t-4 & A_t-1 & A_t-2 & A_t-3 & A_t-4 \\
\hline MAE & 7 & 7.2 & 7.2 & 7.7 & 3.4 & 3.4 & 3.7 & 3.7 \\
\hline MAPE & 12.7 & 13.2 & 13.2 & 14.2 & 19.6 & 20 & 21.3 & 21.3 \\
\hline RMSE & 8.7 & 9.1 & 9 & 9.6 & 4.4 & 4.4 & 4.7 & 4.7 \\
\hline
\end{tabular}

Prefix 'E' represent elderly; prefix 'A' represent adults.

Table 2: Comparison of performance of the 3 models

\begin{tabular}{|c|c|c|c|}
\hline & A-ARIMA & VAR & $\begin{array}{c}\text { Seasonal } \\
\text { VAR }\end{array}$ \\
\hline MAE & 9.2 & 9.8 & 9.8 \\
\hline MAPE & 12.8 & 12.8 & 12.8 \\
\hline RMSE & 11.1 & 12.1 & 11.9 \\
\hline
\end{tabular}

